\title{
Molecular Dynamics Simulation Study on a Monolayer of Half [2]Rotaxane Self- Assembled on Au(111).
}

\author{
Yun Hee Jang, Seung Soon Jang, and William A. Goddard, III* \\ Materials and Process Simulation Center, Beckman Institute (139-74) \\ California Institute of Technology, Pasadena, California 91125
}

\section{Supporting Information}

\section{S1. Force field}

We used a force field (FF) based on the Dreiding FF. ${ }^{1}$ The functional forms and parameters are given in Tables S1-S2 and Figure S1.

This FF excludes nonbonding interactions between bonded atoms (1,2-pairs) and atoms bonded to a common atom (1,3pairs). Except for the Au-S pair for which the van der Waals (vdW) parameters were specified explicitly, the off-diagonal van der Waals (vdW) parameters (between different atom types) were determined from the diagonal vdW parameters (between the same atoms types) by the standard combination rule. ${ }^{1}$

For the angle bending term for $\mathrm{PF}_{6}$, a cosine function (with the periodicity of 4), instead of the usual harmonic function, was used to keep it octahedral.

The torsion term for the $\mathrm{O}-\mathrm{C}\left(s p^{3}\right)-\mathrm{C}\left(s p^{3}\right)-\mathrm{O}$ bond in the ethylene oxide linker between TTF and DNP was fit to reproduce the QM (B3LYP/6-31G**) potential energy curve calculated along the torsion scan with respect to the central bond of $\mathrm{CH}_{3} \mathrm{OCH}_{2} \mathrm{CH}_{2} \mathrm{OCH}_{3}$ (Figure $\mathrm{S} 2$ ).

Table S1. Functional forms used in the force field. ${ }^{a}$

$$
\begin{aligned}
& E=E^{\text {nonbond }}+E^{\text {valence }} \\
& E^{\text {nonbond }}=E^{\text {coulomb }}+E^{v d W} \\
& E^{\text {valence }}=E^{\text {bond }}+E^{\text {angle }}+E^{\text {torsion }}+E^{\text {inversion }} \\
& E_{i j}^{\text {coulomb }}(R)=C_{0} \frac{q_{i} q_{j}}{\varepsilon R_{i j}} \\
& E_{i j}^{v d W}(R)=D_{0}\left\{\left[\left(\frac{6}{\varsigma-6}\right) e^{\varsigma\left(1-\frac{R}{R_{0}}\right)}\right]-\left[\left(\frac{\varsigma}{\varsigma-6}\right)\left(\frac{R_{0}}{R}\right)^{6}\right]\right\} \\
& E^{\text {bond }}(R)=\frac{1}{2} K_{R}\left(R-R_{0}\right)^{2} \\
& E^{\text {angle }}(\theta)=\frac{1}{2} \frac{K_{\theta}}{\sin ^{2} \theta_{0}}\left(\cos \theta-\cos \theta_{0}\right)^{2} \\
& E_{O h}^{\text {angle }}(\theta)=\frac{1}{2} \frac{K_{\theta}}{N^{2}}\left[1-B(-1)^{N} \cos (N \theta)\right](N=4) \\
& E^{\text {torsion }}(\varphi)=\frac{1}{2} \sum_{n} K_{\varphi, n}[1-d \cos (n \varphi)] \\
& E^{\text {inversion }}(\omega)=\frac{1}{2} \frac{K_{\omega}}{\sin ^{2} \omega_{0}}\left(\cos \omega-\cos \omega_{0}\right)^{2}
\end{aligned}
$$

${ }^{\text {a }}$ The constants in $E^{\text {Coulomb }}$ are the dielectric constant $(\varepsilon=1)$ and $C_{0}=$ 332.0637 (the unit conversion factor when atomic charges are in electron units $(|\mathrm{e}|)$, the distance $R$ is in $\AA$, and $E^{C o u l o m b}$ is in $\left.\mathrm{kcal} / \mathrm{mol}\right)$.

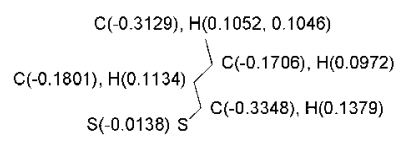

(a) butanethiolate (BuS?)

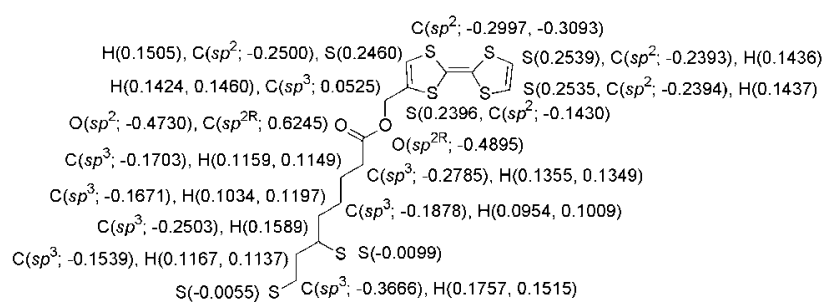

(b) thioctate-TTF

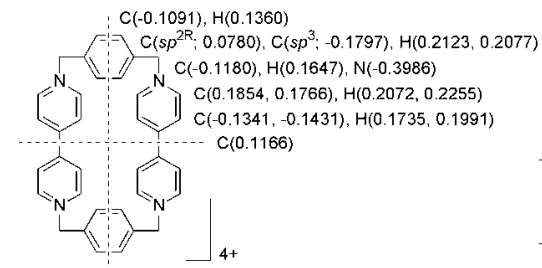

(c) $\mathrm{CBPQT}^{4+}$

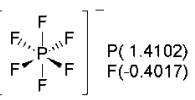

(d) $\mathrm{PF}_{6}^{-}$

Figure S1. Atomic charges $\left(q_{\mathrm{i}}\right)$ used in the force field. They were calculated quantum mechanically at the B3LYP/6-31G** level on the final geometry of each component optimized separately at the same level of theory using Jaguar v4.2. The geometries and charges of BuS? (doublet) and thioctate-TTF (broken S-S; triplet) were calculated using the unrestricted DFT.

Table S2. Force field parameters.

\begin{tabular}{llll}
\hline$E^{\mathrm{vdW}}$ (diagonal) & $R_{\mathrm{o}}^{\mathrm{a}}$ & $D_{\mathrm{o}}^{\mathrm{b}}$ & $\zeta$ \\
$\mathrm{Au}$ & 2.993 & 3.0346 & 12 \\
$\mathrm{~S}$ & 4.03 & 0.344 & 12 \\
$\mathrm{C}\left(\mathrm{sp}^{3}\right)$ & 3.8983 & 0.0951 & 12 \\
$\mathrm{H}$ & 3.195 & 0.016 & 11.8 \\
$\mathrm{C}\left(\mathrm{sp}^{2}\right), \mathrm{C}\left(\mathrm{sp}^{2 \mathrm{R}}\right)$ & 3.8837 & 0.0844 & 12 \\
$\mathrm{O}\left(\mathrm{sp}^{2}\right), \mathrm{O}\left(\mathrm{sp}^{2 \mathrm{R}}\right)$ & 3.4046 & 0.0957 & 13.483 \\
$\mathrm{~N}\left(\mathrm{sp}^{2}\right), \mathrm{N}\left(\mathrm{sp}^{2 \mathrm{R}}\right)$ & 3.6621 & 0.0774 & 13.843 \\
$\mathrm{P}$ & 4.15 & 0.32 & 12 \\
$\mathrm{~F}$ & 3.3825 & 0.05092 & 15 \\
$E^{\mathrm{vdW}}($ off-diagonal $)$ & $R_{\mathrm{o}}^{\mathrm{a}}$ & $D_{\mathrm{o}}^{\mathrm{b}}$ & $\zeta$ \\
$\mathrm{Au}-\mathrm{S}$ & 2.879 & 4.2048 & 12 \\
$E^{\mathrm{bond}}$ & $R_{\mathrm{o}}^{\mathrm{a}}$ & $K_{\mathrm{b}}^{\mathrm{c}}$ &
\end{tabular}




\begin{tabular}{|c|c|c|c|}
\hline $\mathrm{S}-\mathrm{C}\left(\mathrm{sp}^{3}\right)$ & 1.8 & 700 & \\
\hline$C\left(\mathrm{sp}^{3}\right)-\mathrm{C}\left(\mathrm{sp}^{3}\right)$ & 1.53 & 700 & \\
\hline $\mathrm{C}\left(\mathrm{sp}^{3}\right)-\mathrm{H}$ & 1.09 & 700 & \\
\hline $\mathrm{S}-\mathrm{C}\left(\mathrm{sp}^{2}\right)$ & 1.7 & 700 & \\
\hline $\mathrm{C}\left(\mathrm{sp}^{2}\right)-\mathrm{C}\left(\mathrm{sp}^{2}\right)$ & 1.33 & 1400 & \\
\hline $\mathrm{C}\left(\mathrm{sp}^{3}\right)-\mathrm{C}\left(\mathrm{sp}^{2}\right)$ & 1.43 & 700 & \\
\hline $\mathrm{C}\left(\mathrm{sp}^{3}\right)-\mathrm{C}\left(\mathrm{sp}^{2 \mathrm{R}}\right)$ & 1.46 & 700 & \\
\hline $\mathrm{O}\left(\mathrm{sp}^{2}\right)-\mathrm{C}\left(\mathrm{sp}^{2 \mathrm{R}}\right)$ & 1.25 & 1400 & \\
\hline $\mathrm{O}\left(\mathrm{sp}^{2 \mathrm{R}}\right)-\mathrm{C}\left(\mathrm{sp}^{2 \mathrm{R}}\right)$ & 1.35 & 1050 & \\
\hline $\mathrm{C}\left(\mathrm{sp}^{2 \mathrm{R}}\right)-\mathrm{H}$ & 1.02 & 700 & \\
\hline$C\left(s^{2 R}\right)-C\left(s p^{2 R}\right)$ & 1.39 & 1050 & \\
\hline $\mathrm{N}\left(\mathrm{sp}^{2 \mathrm{R}}\right)-\mathrm{C}\left(\mathrm{sp}^{2 \mathrm{R}}\right)$ & 1.34 & 1050 & \\
\hline $\mathrm{N}\left(\mathrm{sp}^{2 \mathrm{R}}\right)-\mathrm{C}\left(\mathrm{sp}^{3}\right)$ & 1.41 & 700 & \\
\hline P-F & 1.6378 & 474.326 & \\
\hline$E^{\text {angle }}$ & $\theta_{\mathrm{o}}^{\mathrm{d}}$ & $K_{\theta}^{\mathrm{b}}$ & \\
\hline $\mathrm{X}-\mathrm{C}\left(\mathrm{sp}^{3}\right)-\mathrm{X}$ & 109.47 & 100 & \\
\hline $\mathrm{X}-\mathrm{C}\left(\mathrm{sp}^{2,2 \mathrm{R}}\right)-\mathrm{X}$ & 120 & 100 & \\
\hline $\mathrm{X}-\mathrm{S}-\mathrm{X}$ & 109.47 & 350 & \\
\hline $\mathrm{X}-\mathrm{O}\left(\mathrm{sp}^{2 \mathrm{R}}\right)-\mathrm{X}$ & 120 & 100 & \\
\hline$X-N\left(s p^{2 R}\right)-X$ & 120 & 100 & \\
\hline$E^{\text {angle }}\left(O_{\mathrm{h}}\right)$ & $N$ & $K_{\theta}^{\mathrm{b}}$ & $B$ \\
\hline F-P-F (in $\mathrm{PF}_{6}^{-}$) & 4 & 132.241 & +1 \\
\hline$E^{\text {torsion }}$ & $n$ & $K_{\varphi}{ }^{\mathrm{b}}$ & $d$ \\
\hline $\mathrm{X}-\mathrm{C}\left(\mathrm{sp}^{3}\right)-\mathrm{C}\left(\mathrm{sp}^{3}\right)-\mathrm{X}$ & 3 & 2.0 & -1 \\
\hline \multirow[t]{3}{*}{$\mathrm{O}-\mathrm{C}\left(\mathrm{sp}^{3}\right)-\mathrm{C}\left(\mathrm{sp}^{3}\right)-\mathrm{O}$} & 1 & 25.5 & +1 \\
\hline & 2 & 16.5 & -1 \\
\hline & 3 & 9.75 & +1 \\
\hline $\mathrm{X}-\mathrm{C}\left(\mathrm{sp}^{2}\right)-\mathrm{C}\left(\mathrm{sp}^{3}\right)-\mathrm{X}$ & 3 & 2.0 & -1 \\
\hline$O\left(s p^{2}\right)-C\left(s p^{2}\right)-C\left(s p^{3}\right)-X$ & 6 & 1.0 & +1 \\
\hline$C\left(s^{2}\right)-O\left(s p^{2}\right)-C\left(s p^{3}\right)-X$ & 6 & 1.0 & +1 \\
\hline $\mathrm{X}-\mathrm{C}\left(\mathrm{sp}^{2}\right)-\mathrm{C}\left(\mathrm{sp}^{2}\right)-\mathrm{X}$ & 2 & 45.0 & +1 \\
\hline$X-C\left(s p^{2 R}\right)-C\left(s p^{2 R}\right)-X$ & 2 & 25.0 & +1 \\
\hline $\mathrm{X}-\mathrm{O}\left(\mathrm{sp}^{2}\right)-\mathrm{C}\left(\mathrm{sp}^{2}\right)-\mathrm{X}$ & 2 & 25.0 & +1 \\
\hline $\mathrm{X}-\mathrm{O}\left(\mathrm{sp}^{2}\right)-\mathrm{C}\left(\mathrm{sp}^{3}\right)-\mathrm{X}$ & 3 & 2.0 & -1 \\
\hline $\mathrm{X}-\mathrm{N}\left(\mathrm{sp}^{2}\right)-\mathrm{C}\left(\mathrm{sp}^{3}\right)-\mathrm{X}$ & 3 & 2.0 & -1 \\
\hline $\mathrm{X}-\mathrm{N}\left(\mathrm{sp}^{2}\right)-\mathrm{C}\left(\mathrm{sp}^{2}\right)-\mathrm{X}$ & 2 & 25.0 & +1 \\
\hline$E^{\text {invesion }}$ & $\omega_{0}^{\mathrm{d}}$ & $K_{\omega}^{\mathrm{b}}$ & \\
\hline$C\left(\mathrm{sp}^{2,2 \mathrm{R}}\right)-\mathrm{X}-\mathrm{X}-\mathrm{X}$ & 0.0 & 40.0 & \\
\hline $\mathrm{N}\left(\mathrm{sp}^{2,2 \mathrm{R}}\right)-\mathrm{X}-\mathrm{X}-\mathrm{X}$ & 0.0 & 40.0 & \\
\hline
\end{tabular}

This set of FF turned out to be very good $(\Delta<5 \%)$ in reproducing the density of relevant crystals containing $\mathrm{CBPQT}^{4+}$ (or $\mathrm{PQT}^{2+}$, a fraction of $\mathrm{CBPQT}$ ), $\mathrm{PF}_{6}^{-}, \mathrm{TTF}$, and/or $\mathrm{CH}_{3} \mathrm{CN}$ units (Figure S3) from the 1-ns NPT MD simulation at corresponding temperatures (Table S3).

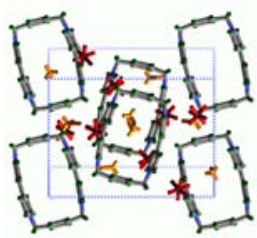

(a) VAFRID

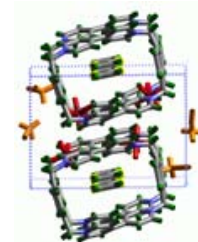

(b) VOLMEO

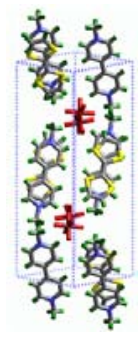

(c) UBULUY

Figure S3. Crystals of CBPQT (PQT), $\mathrm{PF}_{6}, \mathrm{TTF}$, and $\mathrm{CH}_{3} \mathrm{CN}^{2-5}$

Table S3. Crystal structure data. Exeriment ${ }^{2-5}$ versus calculation.

\begin{tabular}{|c|c|c|c|c|c|}
\hline & Code & Contents & $\mathrm{T} / \mathrm{K}$ & $\rho_{\text {expt }}$ & $\rho_{\mathrm{MD}}(\Delta)$ \\
\hline (a) & VAFRID & $\begin{array}{c}\text { CBPQT, } \mathrm{PF}_{6}, \\
\mathrm{CH}_{3} \mathrm{CN}\end{array}$ & 293 & $\begin{array}{l}1.500 \\
\mathrm{~g} / \mathrm{cm}^{3}\end{array}$ & $\begin{array}{c}1.432 \\
(4.7 \%)\end{array}$ \\
\hline (b) & VOLMEO & $\begin{array}{l}\text { CBPQT, } \mathrm{PF}_{6}, \\
\mathrm{TTF}, \mathrm{CH}_{3} \mathrm{CN}\end{array}$ & 295 & $\begin{array}{l}1.546 \\
\mathrm{~g} / \mathrm{cm}^{3}\end{array}$ & $\begin{array}{l}1.594 \\
(3 \%)\end{array}$ \\
\hline (c) & UBULUY & $\mathrm{PQT}, \mathrm{PF}_{6}, \mathrm{TTF}$, & 160 & $\begin{array}{l}1.789 \\
\mathrm{~g} / \mathrm{cm}^{3}\end{array}$ & $\begin{array}{c}1.801 \\
(0.67 \%)\end{array}$ \\
\hline
\end{tabular}

The MD simulation at $298 \mathrm{~K}$ using our set of FF gives the binding energy of $\sim 3 \mathrm{kcal} / \mathrm{mol}$ between $(\mathrm{CBPQT})\left(\mathrm{PF}_{6}\right)_{4}$ and $\mathrm{TTF}$, which is in the range of the experimental estimates for the free energy of complexation of $(\mathrm{TTF})(\mathrm{CBPQT})\left(\mathrm{PF}_{6}\right)_{4}$ complex $(5.3-5.5 \mathrm{kcal} / \mathrm{mol}){ }^{6-8}$

\section{S3. Scheme-dependence}

Even though long MD simulations of $1 \mathrm{~ns}$ were employed to estimate the coverage-dependence of packing energy in order to ensure enough equilibration and sampling (Figure 4 and Figure S4c), shorter MD simulations (such as $100 \mathrm{ps)} \mathrm{or} \mathrm{even} \mathrm{the}$ minimization only give the same trend of the results (Figure S4).
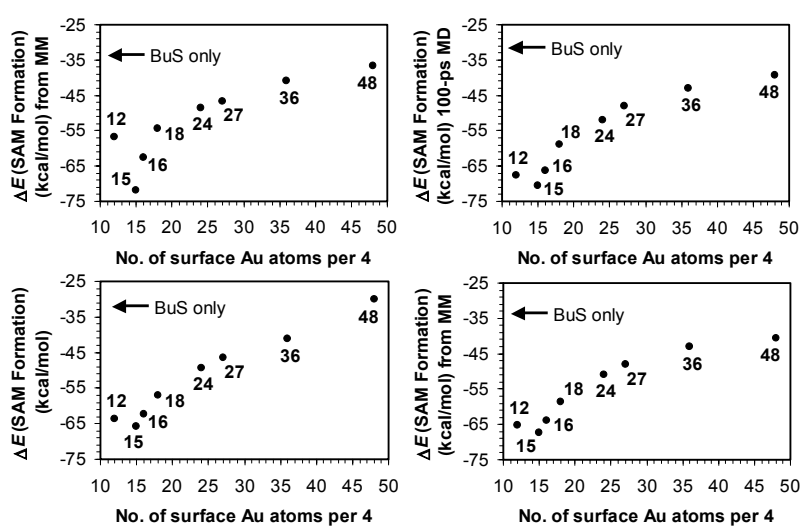

Figure S4. Coverage-dependent packing energy of 4 (a) from the initial minimizations, (b) from shorter (100-ps) NVT MD simulations at $298 \mathrm{~K}$, (c) from longer (1-ns) simulations, and (d) from the final minimizations after the 1-ns simulation ( $300 \mathrm{ps}$ for the $\mathbf{1 / 4 8}$ case).

\section{S2. Force field validation}

\section{References}


(1) Mayo, S. L.; Olafson, B. D.; Goddard, W. A., III J. Phys. Chem. 1990, 94, 8897-8909.

(2) Odell, B.; Reddington, M. V.; Slawin, A. M. Z.; Spencer, N.; Stoddart, J. F.; Williams, D. J. Angew. Chem. Int. Ed. Engl. 1988, 27.

(3) Anelli, P. L.; Ashton, P. R.; Ballardini, R.; Balzani, V.; Delgado, M.; Gandolfi, M. T.; Goodnow, T. T; Kaifer, A. E.; Philp, D. Pietraszkiewicz, M.; Prodi, L.; Reddington, M. V.; Slawin, A. M. Z.; Spencer, N.; Stoddart, J. F.; Vicent, C.; Williams, D. J J. Am. Chem. Soc. 1992, 114, 193-218.

(4) Philp, D.; Slawin, A. M. Z.; Spencer, N.; Stoddart, J. F.; Williams, D. J. J. Chem. Soc., Chem. Commun. 1991, 1584-1586.

(5) Cooke, G.; de Cremiers, H. A.; Duclairoir, F. M. A.; Gray, M.; Vaqueiro, P.; Powell, A. V.; Rosair, G.; Rotello, V. M. Tetrahed. Lett. 2001, 42, 5089-5091.

(6) Anelli, P. -L.; Asakawa, M.; Ashton, P. R.; Bissell, R. A.; Clavier, G.; Gorski, R.; Kaifer, A. E.; Langford, S. J.; Mattersteig, G.; Menzer, S.; Philp, D.; Slawin, A. M. Z.; Spencer, N.; Stoddart, J. F.; Tolley, M. S.; Williams, D. J. Chem. Eur. J. 1997, 3, 1113-1135.

(7) Pease, A. R.; Jeppesen, J. O.; Stoddart, J. F.; Luo, Y.; Collier, C. P.; Heath, J. R. Acc. Chem. Res. 2001, 34, 433-444.

(8) Bryce, M. R.; Cooke, G.; Duclairoir, F. M. A.; Rotello, V. M. Tetrahed. Lett. 2001, 42, 1143-1145. 